BULL. AUSTRAL. MATH. SOC.

VOL. $23(1981), 1-4$.

\title{
ON NORMAL SUBSPACES
}

\section{D.B. Gauld, I.L. Reilly and M.K. Vamanamurthy}

\begin{abstract}
In this paper the anti-normal property is studied. A space is anti-normal if its only normal subspaces are those whose cardinalities require them to be normal. It is shown that every topological space of at least four elements contains a normal three point subspace from which it follows that there is only one non-trivial anti-normal space.
\end{abstract}

This paper answers the question raised in [2], concerning the characterization of the anti-normal spaces. In so doing, it provides yet another topological feature which distinguishes normality from every other separation property.

In this note, we particularize the discussion of topological antiproperties to the class of anti-normal spaces. The general discussion is given in the papers of Bankston [1] and Reilly and Vamanamurthy [2], and we follow their set-theoretic and notational conventions.

A topological space $X$ is anti-normal if and only if the only normal subspaces of $X$ are those whose cardinalities require them to be normal. The spectrum of a topological class $K$, is the class of cardinal numbers $K$ such that any topology on a set of power $K$ lies in $K$. In [2] it was shown that $\operatorname{spec}($ normal) $=\{0,1,2\}$, but the authors were unable to obtain a characterization of the class of anti-normal spaces. Here, we are able to give a rather surprising characterization of the anti-normal spaces, especially in view of the results for other classes defined by separation properties in $[2, \$ 2]$. Indeed, by proving that any topological

Received 16 July 1980. 
space having at least four elements contains a normal three point subspace, we can show that there is only one non-trivial anti-normal space, up to homeomor phism.

Our first proposition is part of the folklore.

PROPOSITION 1. Let $X=\{a, b, c\}$ be a three point set. There are nine homeomorphism classes of topologies on $X$, of which only one is not normal. This topology is characterized by one open and not closed singleton, and two closed and not open singletons.

Proof. The homeomorphism classes are as follows:

(i) discrete, one member,

(ii) indiscrete, one member,

(iii) $\{\varnothing, X,\{a\}\}$, three members,

(iv) $\{\varnothing, X,\{a, b\}\}$, three members,

(v) $\{\varnothing, X,\{a\},\{a, b\}\}$, six members,

(vi) $\{\emptyset, X,\{a\},\{b, c\}\}$, three members,

(vii) $\{\emptyset, X,\{a\},\{b\},\{a, b\}\}$, three members,

(viii) $\{\varnothing, X,\{a\},\{b\},\{a, b\},\{b, c\}\}$, six members,

(ix) $\{\varnothing, X,\{a\},\{a, b\},\{a, c\}\}$, three members.

We observe that the classes (i), (ii) and (vi) are regular and normal, the classes (iii), (iv), (v), (vii) and (viii) are normal but not $R_{0}$, and class (ix) is neither normal nor $R_{0}$. (A topological space $(X, T)$ is $R_{0}$ if and only if $x \in U \in T$ implies $T \operatorname{cl}\{x\} \subset U$.)

PROPOSITION 2. Spectrum (normal) $=\{0,1,2\}=3$.

Proof. Any topology on a set of at most two points is normal. From Proposition 1, we have a three point non-normal space. If $X$ has more than three points, let $X=\{a, b, c\} \cup E$, where the union is disjoint. Then $(X, T)$ is not normal where

$$
T=\{\varnothing, X,\{a\} \cup E,\{a, b\} \cup E,\{a, c\} \cup E\} .
$$

THEOREM 1. Let $X$ be a topological space having at least four elements. Then $X$ contains a normal three point subspace. 
Proof. It suffices to consider the case where $X$ has exactly four points; otherwise replace $X$ by any four point subspace. Suppose $X=\{a, b, c, d\}$. We consider three cases.

I. Suppose $X$ has a three point open subset, say $\{a, b, c\}$. If $\{a, b, c\}$ is normal, we are through. Otherwise, it is non-normal, so by Proposition 1 (ix), two doubleton subsets of $\{a, b, c\}$ are open in $\{a, b, c\}$, say $\{a, b\}$ and $\{a, c\}$. Since $\{a, b, c\}$ is open in $X$, $\{a, b\}$ and $\{a, c\}$ are open in $X$. Hence, $\{b\}$ and $\{c\}$ are open in $\{b, c, d\}$, and thus $\{b, c, d\}$ is a normal three point subspace of $X$.

II. Suppose $X$ has a two point open subset, say $\{a, b\}$. If $\{a, b, c\}$ is normal, we are through. Otherwise, it is non-normal, so by Proposition $1(i x)$, at least one of $\{a, c\}$ and $\{b, c\}$ is open in $\{a, b, c\}$, say $\{a, c\}$. Thus either $\{a, c\}$ or $\{a, c, d\}$ is open in $X$. In either case we obtain a three point open subset of $X$, namely $\{a, b, c\}=\{a, b\} \cup\{a, c\}$ in the former, and $\{a, c, d\}$ in the latter. Thus case I contains case II.

III. Finally suppose that $X$ contains neither two point nor three point open subsets. Hence, either $X$ is indiscrete or $X$ contains only one proper open set and this open set is a singleton, say $\{a\}$. In either of these cases $X$ contains an indiscrete, and hence normal, three point subspace, for example, $\{b, c, d\}$.

THEOREM 2. There is only one non-trivial anti-normal space (up to homeomorphism), namely the three point non-normal space.

Proof. By Proposition 2, the three point non-normal space is antinormal. If $X$ has more than three points, then by Theorem 1, it has a normal three point subspace, and hence $X$ is not anti-normal.

\section{References}

[1] Paul Bankston, "The total negation of a topological property", Illinois J. Math. 23 (1979), 241-252. 
[2] I.L. Reilly and M.K. Vamanamurthy, "Some topological anti-properties", IZZinois J. Math. (to appear).

Department of Mathematics,

University of Auckland,

Private Bag,

Auckl and,

New Zealand. 